Provided for non-commercial research and education use. Not for reproduction, distribution or commercial use.

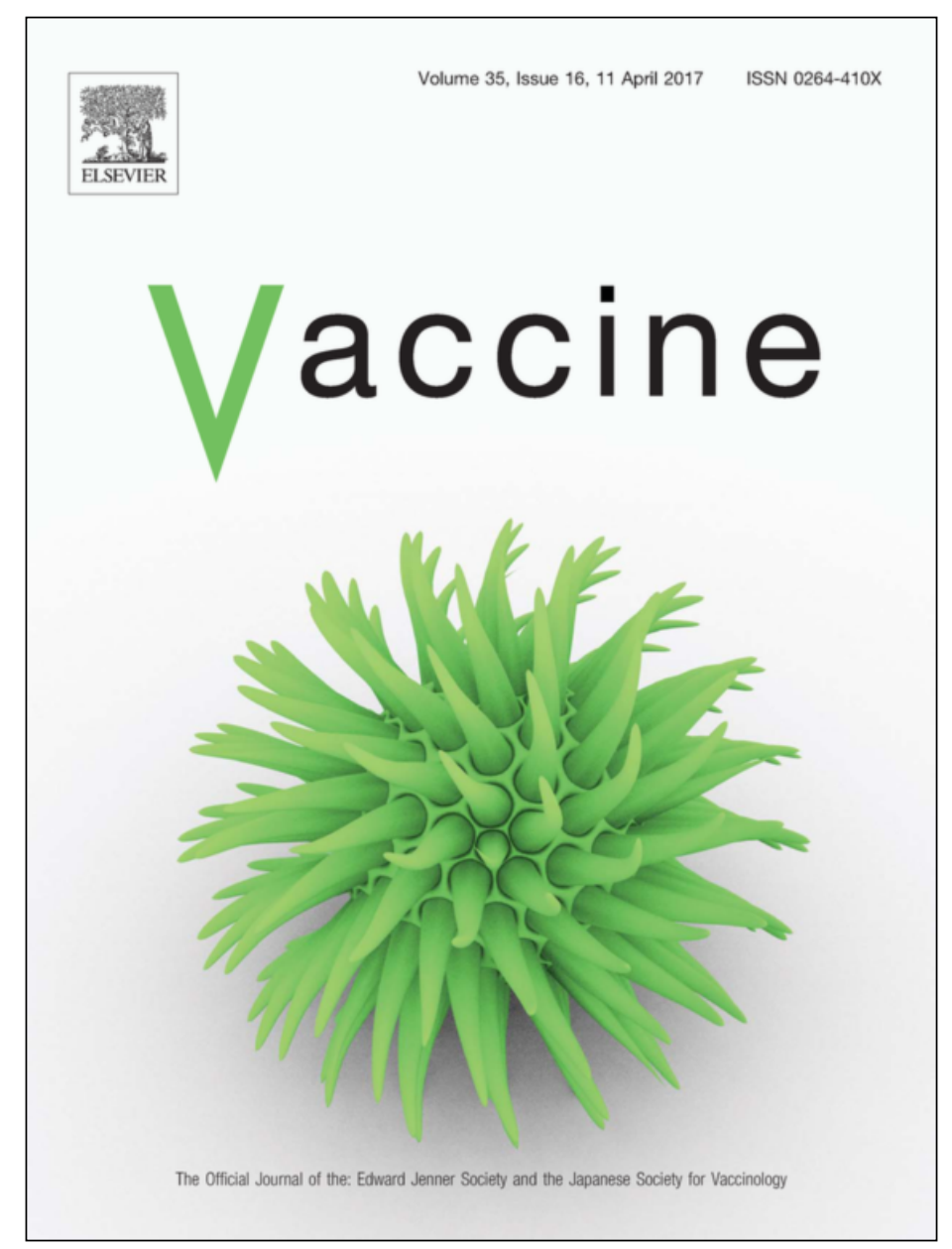

This article appeared in a journal published by Elsevier. The attached copy is furnished to the author for internal non-commercial research and education use, including for instruction at the author's institution and sharing with colleagues.

Other uses, including reproduction and distribution, or selling or licensing copies, or posting to personal, institutional or third party websites are prohibited.

In most cases authors are permitted to post their version of the article (e.g. in Word or Tex form) to their personal website or institutional repository. Authors requiring further information regarding Elsevier's archiving and manuscript policies are encouraged to visit:

http://www.elsevier.com/authorsrights 
Review

\title{
Facilitators and barriers of parental attitudes and beliefs toward school- located influenza vaccination in the United States: Systematic review
}

\author{
Gloria J. Kang, Rachel K. Culp, Kaja M. Abbas* \\ Department of Population Health Sciences, Virginia-Maryland College of Veterinary Medicine, Virginia Tech, USA
}

\section{A R T I C L E I N F O}

\section{Article history:}

Received 8 December 2016

Received in revised form 1 March 2017

Accepted 7 March 2017

Available online 18 March 2017

\section{Keywords:}

Influenza

School-located vaccination

Parental attitudes

Parental beliefs

United States

\begin{abstract}
A B S T R A C T
The study objective was to identify facilitators and barriers of parental attitudes and beliefs toward school-located influenza vaccination in the United States. In 2009, the Advisory Committee on Immunization Practices of the Centers for Disease Control and Prevention expanded their recommendations for influenza vaccination to include school-aged children. We conducted a systematic review of studies focused on facilitators and barriers of parental attitudes toward school-located influenza vaccination in the United States from 1990 to 2016 . We reviewed 11 articles by use of the PRISMA (Preferred Reporting Items for Systematic Reviews and Meta-Analyses) framework. Facilitators were free/low cost vaccination; having belief in vaccine efficacy, influenza severity, and susceptibility; belief that vaccination is beneficial, important, and a social norm; perception of school setting advantages; trust; and parental presence. Barriers were cost; concerns regarding vaccine safety, efficacy, equipment sterility, and adverse effects; perception of school setting barriers; negative physician advice of contraindications; distrust in vaccines and school-located vaccination programs; and health information privacy concerns. We identified the facilitators and barriers of parental attitudes and beliefs toward school-located influenza vaccination to assist in the evidence-based design and implementation of influenza vaccination programs targeted for children in the United States and to improve influenza vaccination coverage for populationwide health benefits.
\end{abstract}

(c) 2017 Elsevier Ltd. All rights reserved.

\section{Contents}

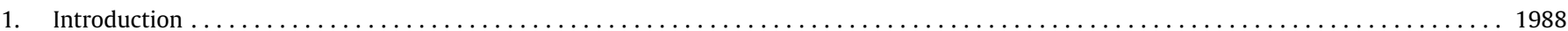

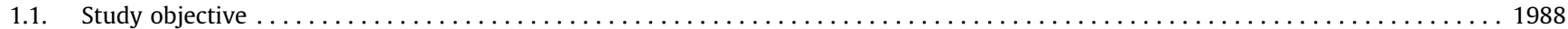

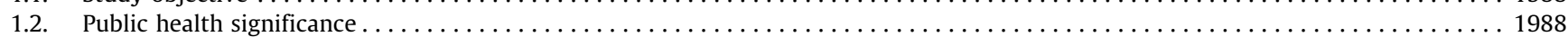

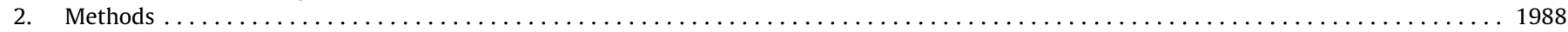

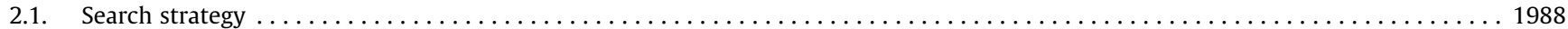

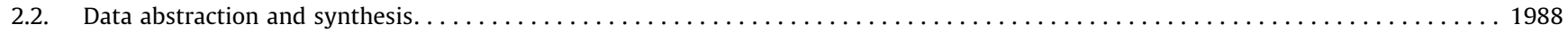

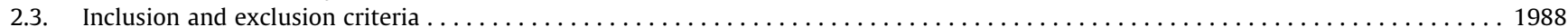

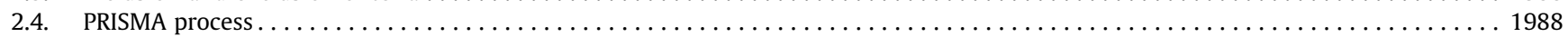

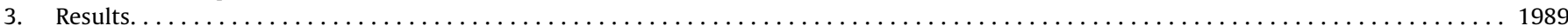

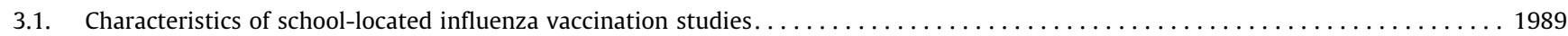

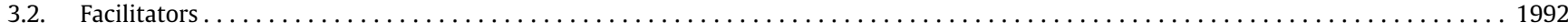

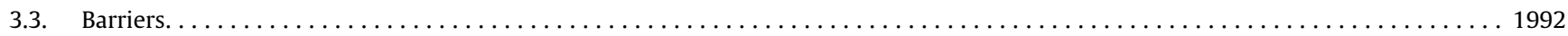

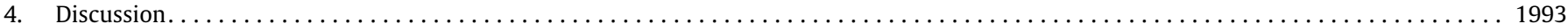

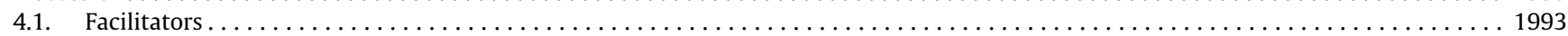

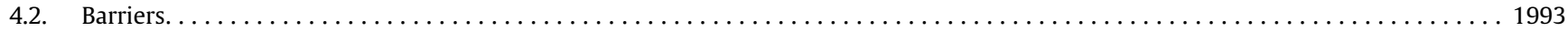

4.3. School-located influenza vaccination in school-based clinics versus delivery by external agencies $\ldots \ldots \ldots \ldots \ldots \ldots \ldots \ldots \ldots \ldots$

\footnotetext{
* Corresponding author at: Department of Population Sciences, Virginia Tech, 205 Duck Pond Drive (MC 0395), Blacksburg, VA 24061, USA.

E-mail address: kaja.abbas@vt.edu (K.M. Abbas).
} 


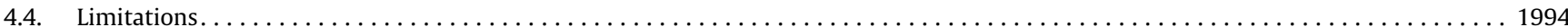

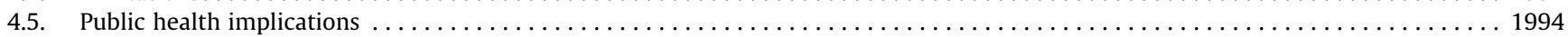

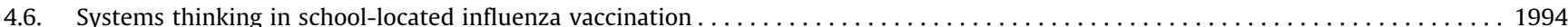

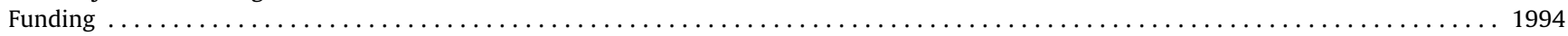

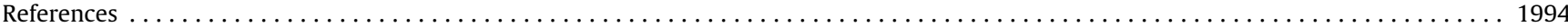

\section{Introduction}

School-based health interventions have been implemented throughout the United States, with most school-based health clinics offering vaccination services to the general school community. In contrast, school-located vaccination (SLV) programs, and specifically school-located influenza vaccination (SLIV), are dedicated programs for targeted vaccination of school-aged children [1,2]. SLV programs have been adopted worldwide in countries such as Canada [3], the United Kingdom [4], and Australia [5]. While less common in the United States, school-located programs for influenza vaccination have shown success statewide in Hawaii [6] and in pilot studies in Tennessee [7] and Maryland communities [8].

Since the 2009 H1N1 influenza pandemic, SLIV programs have gained significant public health interest [1] for improving adolescent vaccination rates in non-clinical settings [9-12], potentially reducing emergency care visits for influenza-like illnesses, lowering community influenza risk, decreasing laboratory-confirmed cases, and improving school attendance $[13,14]$. In a modeling study by Weycker et al., authors found that vaccinating $20 \%$ of children in the United States decreased the total number of influenza cases in the total population by $46 \%$, along with similar decreases in influenza-related mortality and economic costs [15]. However, because SLIV participation ultimately depends on parental consent, there is a need for enhanced understanding of parental attitudes and beliefs regarding SLIV in order to improve influenza vaccination rates among school children in the United States.

\subsection{Study objective}

Our study objective was to identify the facilitators and barriers of parental attitudes and beliefs toward school-located influenza vaccination in the United States, thereby assisting in the evidence-based design and implementation of current and future influenza vaccination programs targeted for children, by leveraging facilitators and addressing potential barriers of parental consent.

\subsection{Public health significance}

In 2009, the Advisory Committee on Immunization Practices (ACIP) of the Centers for Disease Control and Prevention expanded recommendations for targeted influenza vaccination by including school-aged children in the United States [16]. While this has improved vaccination coverage among children (6 months17 years) from $43.7 \%$ during the 2009-2010 influenza season to $59.3 \%$ during the 2015-2016 season [17], this is below the target of $70 \%$ in the Healthy People 2020 initiative [18].

Despite globally recognized benefits of school-located vaccination, the evidence base for SLIV acceptance in the United States is limited [11,12], with studies focused on clinical aspects of vaccine efficacy [19], program feasibility [20], and population-level benefits [21]. We conducted a systematic review to address this evidence gap to improve influenza vaccination coverage by identifying facilitators and barriers of parental attitudes and beliefs toward school-located influenza vaccination for children in the United States.

\section{Methods}

\subsection{Search strategy}

We conducted our search using PubMed and Web of Science databases for articles written in the English language, published between 01/01/1990 and 10/01/2016, and contained the following the terms: (influenza) AND (vaccine OR vaccination OR immunization) AND (school OR school-located OR school-based) AND (parent OR parental).

\subsection{Data abstraction and synthesis}

The data abstraction and synthesis process were conducted by two authors (GJK and RKC) independently; we resolved discordant decisions through consensus. Data abstraction and synthesis included the following four steps: identification, screening, eligibility, and inclusion. During the identification step, articles were identified using the aforementioned search strategy. During screening, duplicate articles were removed, and the titles and abstracts of the remaining articles were screened to determine relevance to our study objectives. During the eligibility step, article full text was analyzed to further determine relevance to our study objectives and to be used for inclusion.

\subsection{Inclusion and exclusion criteria}

We included articles that focused on childhood/adolescent age groups to target school-aged children in grades PreK-12 which met the following study criteria: (1) conducted qualitative and/or quantitative analysis regarding influenza vaccination for schoolaged children in the United States; and (2) assessed parental factors associated with the acceptance, hesitancy, or refusal of utilizing school-located influenza vaccination for children, including parental knowledge, beliefs, and attitudes. We excluded studies that focused on general vaccine delivery (i.e. non-specific to influenza vaccine), studies of non-explicit parent populations (such as school personnel and health care workers who may also be parents), and studies taking place outside the United States.

\subsection{PRISMA process}

Fig. 1 illustrates the process flow diagram of identification, screening, eligibility, and inclusion of articles for the systematic review, using the PRISMA (Preferred Reporting Items for Systematic Reviews and Meta-Analyses) framework [22]. Eleven articles met our selection criteria for systematic review of facilitators and barriers of parental attitudes and beliefs toward school-located influenza vaccination in the United States. While we have included quantitative metrics of the clinical effect size of statistical association for each of the 11 studies, we have excluded quantitative synthesis using meta-analysis due to the heterogeneity in study design and population sampling of these 11 studies. 


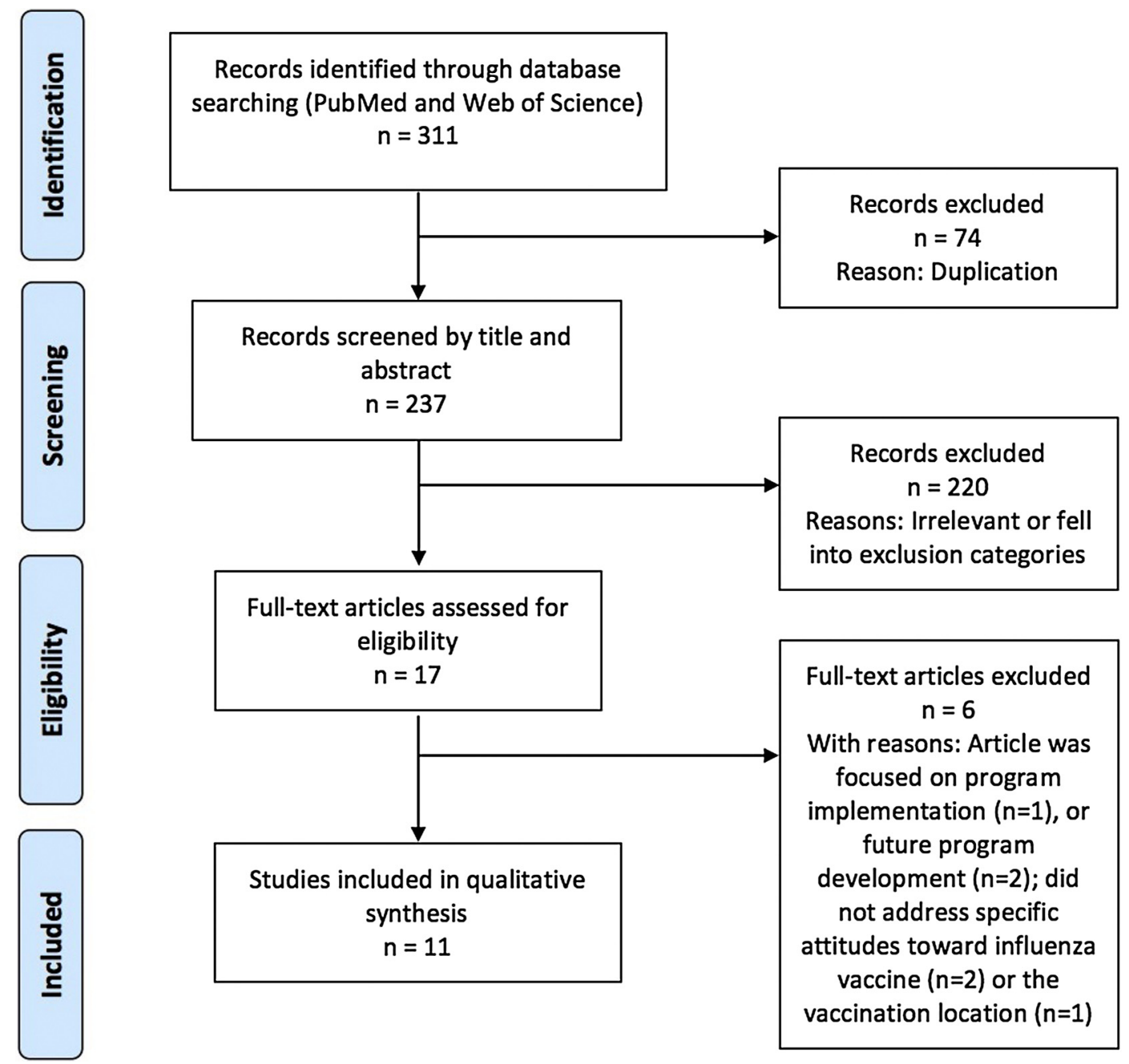

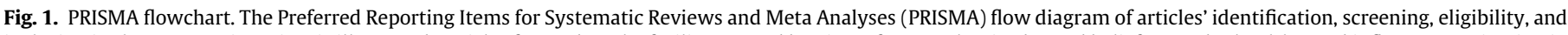

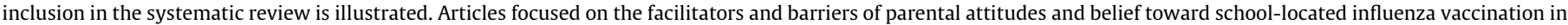
the United States were included, while articles focused on non-influenza vaccination, non-parent populations, and regions outside of United States were excluded.

\section{Results}

\subsection{Characteristics of school-located influenza vaccination studies}

We identified 11 articles focused on school-located influenza vaccination (SLIV) for analysis based on the inclusion and exclusion criteria of our systematic review. Table 1 illustrates the objectives of the 11 studies, SLIV context (hypothetical or actual program context), school settings, geographic area, type of survey and/or focus group, parental sample sizes, and significant inferences regarding parental attitudes and beliefs of SLIV for school-aged children in the United States.

Allison et al. surveyed elementary school parents in Salt Lake City, Utah and found that SLIV programs should address vaccine safety, benefit, cost, and convenience, while promoting vaccination as a social norm [9]. Brown et al. conducted an online survey of a nationally representative sample of parents, whose youngest child was less than 15 years old. While the convenience of SLIV promoted parental acceptance, parents preferred a medical location for proper administration and for care of potential medical needs and side effects. Vaccine safety was a significant barrier to consent [11]. Carpenter et al. briefly surveyed parents of large metropolitan public school system in Knoxville, Tennessee and found that significant barriers to SLIV participation included concerns regarding vaccine adverse effects and vaccine virus transmission to household members with health issues such as asthma [7]. A two-year survey conducted by Cheung et al. in urban elementary schools of Los Angeles County, California found that parents with better understanding of influenza risks and influenza vaccine benefits were more likely to consent to SLIV [23]. Gargano et al. surveyed middle and high school parents in Richmond County, Georgia and found that SLIV acceptance by parents correlated with parental beliefs of influenza vaccination being a social norm and perception of illness severity prevented by vaccination in general [24]. Kelminson et al. conducted a survey of parents in urban/suburban middle schools in Aurora, Colorado and found that belief in vaccine importance was associated with SLIV acceptance; parental absence during vaccination was a major barrier to consent [25]. Kempe et al. conducted a survey of public elementary school parents in a low-income area of Denver, Colorado and found that SLIV was strongly supported by parents due to belief in vaccine efficacy and convenience of a school setting, while the barriers involved concerns regarding vaccine safety and parental absence during vaccination [26].

Focus group discussions of parents and students were conducted by Herbert et al. in a low-income, rural county of Georgia; the barriers of non-participating parents in SLIV involved distrust, suspicions of the vaccination clinic, and the lengthy consent 
Table 1

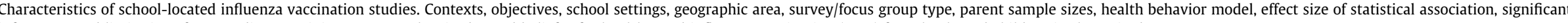
inferences, and limitations from studies pertaining to parental attitudes and beliefs of school-located influenza vaccination (SLIV) for school-aged children in the United States.

\begin{tabular}{|c|c|c|c|c|c|c|c|c|}
\hline Study & Context & Objective & $\begin{array}{l}\text { School setting and } \\
\text { geographic area }\end{array}$ & $\begin{array}{l}\text { Survey/focus } \\
\text { group type and } \\
\text { parental } \\
\text { sample size }\end{array}$ & $\begin{array}{l}\text { Health } \\
\text { behavior } \\
\text { model }\end{array}$ & $\begin{array}{l}\text { Effect size of statistical } \\
\text { association (AOR - Adjusted odds } \\
\text { ratio; RR - Risk ratio; CI - } \\
\text { Confidence interval) }\end{array}$ & Significant inferences & Limitations \\
\hline [9] & $\begin{array}{l}\text { Hypothetical SLIV } \\
\text { program }\end{array}$ & $\begin{array}{l}\text { To identify parental } \\
\text { beliefs, barriers, and } \\
\text { acceptance of SLIV }\end{array}$ & $\begin{array}{l}1 \text { public elementary } \\
\text { school (K-6) in Salt } \\
\text { Lake City, Utah }\end{array}$ & $\begin{array}{l}\text { Cross-sectional } \\
\text { survey of } 259 \\
\text { parents (out of } \\
397 \text { ) }\end{array}$ & $\begin{array}{l}\text { Health Belief } \\
\text { Model }\end{array}$ & $\begin{array}{l}75 \% \text { of parents, including } 59 \% \\
\text { ( } 76 / 129 \text { ) who did not plan to } \\
\text { immunize would consent to SLIV } \\
\text { if offered for free; facilitators } \\
\text { were belief in benefit (AOR: } 6.1 \text {; } \\
95 \% \text { CI: } 2.7-14.0 \text { ), endorsement } \\
\text { of medical setting barriers (AOR: } \\
3.7 ; 95 \% \text { CI: } 1.3-10.3 \text { ), belief that } \\
\text { immunization is a social norm } \\
\text { (AOR: } 3.3 ; 95 \% \text { CI: } 1.4-7.6 \text { ), and } \\
\text { that the child is susceptible to } \\
\text { influenza (AOR: } 2.6 ; 95 \% \text { CI: } 1.2- \\
5.7 \text { ) }\end{array}$ & $\begin{array}{l}\text { SLIV programs should address } \\
\text { barriers of cost and } \\
\text { inconvenience, promote } \\
\text { immunization as a social norm, } \\
\text { and address parental concerns } \\
\text { regarding vaccine safety and } \\
\text { benefit }\end{array}$ & $\begin{array}{l}\text { Most families were Caucasian or } \\
\text { Latino in a single school of a } \\
\text { single season, limiting } \\
\text { generalizability }\end{array}$ \\
\hline [11] & $\begin{array}{l}\text { Hypothetical SLIV } \\
\text { program }\end{array}$ & $\begin{array}{l}\text { To assess the feasibility of } \\
\text { SLIV }\end{array}$ & $\begin{array}{l}\text { Nationally } \\
\text { representative } \\
\text { online sample }\end{array}$ & $\begin{array}{l}\text { Online survey } \\
\text { of } 1088 \text { parents } \\
\text { whose } \\
\text { youngest child } \\
\text { was } \leq 14 \text { years } \\
\text { old }\end{array}$ & $\begin{array}{l}\text { Survey } \\
\text { based on } \\
\text { literature } \\
\text { review of } \\
\text { vaccine } \\
\text { topics, focus } \\
\text { groups, and } \\
\text { pretest } \\
\text { interviews }\end{array}$ & $\begin{array}{l}51 \% \text { of parents would consent to } \\
\text { SLIV; SLIV was more convenient } \\
\text { than the regular location }(42.1 \% \\
\text { of consenting parents versus } \\
19.9 \% \text { of non-consenting } \\
\text { parents, } P<0.001) \text {, however, } \\
\text { regular location was preferred } \\
\text { over SLIV in case of side effects } \\
(46.4 \% \text { vs. } 20.9 \%, P<0.001) \text { and } \\
\text { for proper administration of the } \\
\text { vaccine }(31.0 \% \text { vs. } 21.0 \% \text {, } \\
P<0.001)\end{array}$ & $\begin{array}{l}\text { SLIV convenience promoted } \\
\text { acceptance, but medical location } \\
\text { was preferred for proper } \\
\text { administration and potential } \\
\text { care of side effects; vaccine } \\
\text { safety was a significant barrier }\end{array}$ & $\begin{array}{l}\text { Low response rate limited } \\
\text { generalizability; responses to } \\
\text { hypothetical program do not } \\
\text { reflect likelihood of action }\end{array}$ \\
\hline [7] & $\begin{array}{l}\text { SLIV campaign ( } 2 \\
\text { doses LAIV) } \\
\text { delivered by county } \\
\text { health department }\end{array}$ & $\begin{array}{l}\text { To evaluate the feasibility } \\
\text { and success of SLIV }\end{array}$ & $\begin{array}{l}76 \text { schools (50 } \\
\text { elementary, } 14 \\
\text { middle, } 12 \text { high } \\
\text { schools) from } 1 \\
\text { large metropolitan } \\
\text { public school } \\
\text { system (K-12) in } \\
\text { Knox County, } \\
\text { Tennessee }\end{array}$ & $\begin{array}{l}\text { Brief survey of } \\
1432 \text { parents }\end{array}$ & $\begin{array}{l}\text { Feedback } \\
\text { survey } \\
\text { regarding } \\
\text { SLIV } \\
\text { participation }\end{array}$ & $\begin{array}{l}\text { Non-participation in the } \\
\text { vaccination campaign was } \\
\text { reported by 53\% ( } 34 / 64 \text { ) parents } \\
\text { of black students and } 36 \% \text { ( } 494 / \\
\text { 1339) parents of non- black } \\
\text { students (RR: } 1.44 ; 95 \% \text { CI: } 1.13 \text { - } \\
1.83 \text { ) }\end{array}$ & $\begin{array}{l}\text { Barriers to SLIV participation } \\
\text { included concerns regarding } \\
\text { vaccine adverse effects, } \\
\text { influenza acquisition through } \\
\text { vaccine, and concerns of vaccine } \\
\text { virus transmission to household } \\
\text { members with health issues } \\
\text { such as asthma }\end{array}$ & $\begin{array}{l}\text { Low response rate limited } \\
\text { representativeness }\end{array}$ \\
\hline [23] & $\begin{array}{l}\text { SLIV campaign } \\
\text { delivered by county } \\
\text { public health } \\
\text { department in } 8 \\
\text { schools in year } 1 \text { and } \\
\text { continued in } 4 \\
\text { schools in year } 2\end{array}$ & $\begin{array}{l}\text { To determine predictors of } \\
\text { consent based on parental } \\
\text { attitudes for SLIV }\end{array}$ & $\begin{array}{l}8 \text { urban elementary } \\
\text { schools (PreK-6, } \\
\text { ages 5-13) in 2 } \\
\text { school districts, Los } \\
\text { Angeles County, } \\
\text { California }\end{array}$ & $\begin{array}{l}\text { Survey of } 1259 \\
\text { parents (Year } \\
\text { 1); } 1496 \\
\text { parents (Year } \\
\text { 2) }\end{array}$ & $\begin{array}{l}\text { Health Belief } \\
\text { Model }\end{array}$ & $\begin{array}{l}\text { During } 2009 \text { influenza } \\
\text { pandemic, parents concerned } \\
\text { about influenza severity were } \\
\text { twice as likely to consent for } \\
\text { influenza vaccination compared } \\
\text { to unconcerned parents (OR: } \\
2.04 ; 95 \% \text { CI:1.19-3.51). During } \\
\text { year 2, facilitators of parental } \\
\text { consent were perception of high } \\
\text { susceptibility to influenza (OR: } \\
2.19,95 \% \text { CI: } 1.50-3.19 \text { ) and high } \\
\text { vaccine benefit (OR: } 2.23,95 \% \\
\text { CI:1.47-3.40) }\end{array}$ & $\begin{array}{l}\text { Parents with better } \\
\text { understanding of influenza risks } \\
\text { and influenza vaccine benefits } \\
\text { were more likely to consent for } \\
\text { SLIV }\end{array}$ & $\begin{array}{l}\text { Low response rates suggest } \\
\text { selection bias; higher consent } \\
\text { rates of survey respondents } \\
\text { (compared to school enrollees) } \\
\text { skews responses; respondents } \\
\text { were from low-income, mostly } \\
\text { Hispanic (followed by Asian) } \\
\text { population, limiting } \\
\text { generalizability }\end{array}$ \\
\hline [24] & $\begin{array}{l}\text { Hypothetical } \\
\text { program for } \\
\text { adolescent vaccines } \\
\text { in general; SLV for } \\
\text { influenza had been } \\
\text { previously available }\end{array}$ & $\begin{array}{l}\text { To determine parental } \\
\text { attitudes and acceptance } \\
\text { of school-located } \\
\text { vaccination of middle- and } \\
\text { high school students for } \\
\text { four adolescent }\end{array}$ & $\begin{array}{l}6 \text { middle- and } 5 \\
\text { high schools; urban, } \\
\text { Richmond County, } \\
\text { Georgia }\end{array}$ & $\begin{array}{l}\text { Telephone } \& \\
\text { web survey of } \\
686 \text { parents for } \\
3 \text { years }\end{array}$ & $\begin{array}{l}\text { Health Belief } \\
\text { Model; } \\
\text { Theory of } \\
\text { Reasoned } \\
\text { Action }\end{array}$ & $\begin{array}{l}\text { Facilitators of SLIV were higher } \\
\text { parental perception of benefits } \\
\text { to vaccination (AOR }=1.3 ; 95 \% \mathrm{CI} \\
1.1-1.5 \text { ) and increased social } \\
\text { norms (AOR }=1.44 ; 95 \% \mathrm{CI} 1.12- \\
1.84 \text { ) }\end{array}$ & $\begin{array}{l}\text { SLIV acceptance by parents } \\
\text { correlated with beliefs of } \\
\text { influenza vaccination being a } \\
\text { social norm and perception of } \\
\text { illness severity prevented by } \\
\text { vaccination in general }\end{array}$ & $\begin{array}{l}\text { Low response rate; potential } \\
\text { duplication of responses; single } \\
\text { county population, } \\
\text { predominantly black, low- } \\
\text { income; generalizability is } \\
\text { limited }\end{array}$ \\
\hline
\end{tabular}




\begin{tabular}{|c|c|c|c|c|c|c|c|c|}
\hline Study & Context & Objective & $\begin{array}{l}\text { School setting and } \\
\text { geographic area }\end{array}$ & $\begin{array}{l}\text { Survey/focus } \\
\text { group type and } \\
\text { parental } \\
\text { sample size }\end{array}$ & $\begin{array}{l}\text { Health } \\
\text { behavior } \\
\text { model }\end{array}$ & $\begin{array}{l}\text { Effect size of statistical } \\
\text { association (AOR - Adjusted odds } \\
\text { ratio; RR - Risk ratio; CI - } \\
\text { Confidence interval) }\end{array}$ & Significant inferences & Limitations \\
\hline & & $\begin{array}{l}\text { recommended vaccines, } \\
\text { including influenza } \\
\text { vaccine }\end{array}$ & & & & & & \\
\hline [27] & $\begin{array}{l}\text { Study takes place 2+ } \\
\text { years after } \\
\text { implementation of a } \\
\text { 3-year, multi- } \\
\text { component } \\
\text { influenza } \\
\text { vaccination program } \\
\text { delivered by a } \\
\text { research group }\end{array}$ & $\begin{array}{l}\text { To characterize the } \\
\text { decision-making process } \\
\text { and reasons of parents and } \\
\text { students for participation } \\
\text { in SLIV }\end{array}$ & $\begin{array}{l}\text { Middle and high } \\
\text { school; rural county } \\
\text { in Georgia }\end{array}$ & $\begin{array}{l}\text { Focus group } \\
\text { discussions of } \\
41 \text { parents (and } \\
44 \text { students) }\end{array}$ & $\begin{array}{l}\text { Focus } \\
\text { groups, open } \\
\text { discussion } \\
\text { on attitudes } \\
\text { toward SLIV }\end{array}$ & $\begin{array}{l}\text { Not applicable - no quantitative/ } \\
\text { statistical analysis }\end{array}$ & $\begin{array}{l}\text { Barriers of non-participating } \\
\text { parents of SLIV involved distrust, } \\
\text { suspicions of vaccination clinic, } \\
\text { and the lengthy consent process }\end{array}$ & $\begin{array}{l}\text { Purposive sampling of } \\
\text { respondents from rural Georgia, } \\
\text { predominantly black and low- } \\
\text { income limits generalizability; } \\
\text { focus groups were conducted } \\
\text { over } 2 \text { years after program } \\
\text { implementation, suggesting } \\
\text { recall bias; no demographic or } \\
\text { socioeconomic data collected }\end{array}$ \\
\hline [25] & $\begin{array}{l}\text { Hypothetical SLIV } \\
\text { program }\end{array}$ & $\begin{array}{l}\text { To examine parental } \\
\text { attitudes toward } \\
\text { adolescent vaccination in } \\
\text { school settings, including } \\
\text { influenza vaccine }\end{array}$ & $\begin{array}{l}3 \text { urban/suburban } \\
\text { middle schools } \\
\text { (grade 6) in Aurora, } \\
\text { Colorado }\end{array}$ & $\begin{array}{l}\text { Cross-sectional } \\
\text { mailed survey } \\
\text { of } 500 \text { (out of } \\
\text { 806) parents }\end{array}$ & $\begin{array}{l}\text { Survey } \\
\text { based on } \\
\text { medical } \\
\text { literature }\end{array}$ & $\begin{array}{l}81 \% \text { of parents agreed that SLIV } \\
\text { would be safe and convenient, } \\
\text { however, } 47 \% \text { preferred another } \\
\text { vaccination site }\end{array}$ & $\begin{array}{l}\text { Belief in vaccine importance was } \\
\text { associated with SLIV acceptance } \\
\text { by parents; major barrier was } \\
\text { parental absence during } \\
\text { vaccination }\end{array}$ & $\begin{array}{l}\text { No collection of income data; } \\
\text { possible sampling bias; } \\
\text { responses to hypothetical } \\
\text { program do not reflect likelihood } \\
\text { of action }\end{array}$ \\
\hline [26] & $\begin{array}{l}2 \text { SLIV campaigns } \\
\text { per school delivered } \\
\text { by city public health } \\
\text { department and } \\
\text { community health } \\
\text { services }\end{array}$ & $\begin{array}{l}\text { To assess parental } \\
\text { attitudes and supportive } \\
\text { factors for SLIV of } \\
\text { elementary school } \\
\text { students }\end{array}$ & $\begin{array}{l}20 \text { public } \\
\text { elementary schools } \\
\text { (K-6), low SES; } \\
\text { Denver, Colorado }\end{array}$ & $\begin{array}{l}\text { Survey of } 699 \\
\text { parents }\end{array}$ & $\begin{array}{l}\text { Health Belief } \\
\text { Model }\end{array}$ & $\begin{array}{l}\text { Facilitators were belief in } \\
\text { vaccine efficacy (RR: } 1.49 ; 95 \% \\
\text { CI: } 1.23-1.84 \text { ) and convenience } \\
\text { of school delivery (RR: } 2.37 ; 95 \% \\
\text { CI: } 1.82-3.45 \text { ). Barriers were } \\
\text { safety concerns of influenza } \\
\text { vaccine (RR: } 0.80 ; 95 \% \text { CI: } 0.72- \\
0.88 \text { ) and not wanting their child } \\
\text { vaccinated without a parent (RR: } \\
0.74 ; 95 \% \text { CI: } 0.64-0.83 \text { ) }\end{array}$ & $\begin{array}{l}\text { SLIV was strongly supported by } \\
\text { parents with belief in vaccine } \\
\text { efficacy and convenience of } \\
\text { school setting; barriers involved } \\
\text { concerns about vaccine safety } \\
\text { and parental absence during } \\
\text { vaccination }\end{array}$ & $\begin{array}{l}\text { Low-income, urban population } \\
\text { of mostly ethnic minorities } \\
\text { limits generalizability; survey } \\
\text { conducted during same year as } \\
2009 \text { H1N1 pandemic }\end{array}$ \\
\hline [28] & $\begin{array}{l}\text { Hypothetical SLIV } \\
\text { program }\end{array}$ & $\begin{array}{l}\text { To determine factors } \\
\text { influencing parental } \\
\text { consent of SLIV }\end{array}$ & $\begin{array}{l}1 \text { elementary, } 2 \\
\text { middle, } 3 \text { high } \\
\text { schools in large } \\
\text { urban school } \\
\text { district in Houston, } \\
\text { Texas }\end{array}$ & $\begin{array}{l}37 \text { parents; } 5 \\
\text { focus group } \\
\text { interviews }\end{array}$ & $\begin{array}{l}\text { Questions } \\
\text { were based } \\
\text { on medical } \\
\text { literature }\end{array}$ & $\begin{array}{l}\text { Not applicable - no quantitative/ } \\
\text { statistical analysis }\end{array}$ & $\begin{array}{l}\text { Parental attitudes to SLIV are } \\
\text { impacted by safety and trust } \\
\text { issues regarding vaccines in } \\
\text { general; programs should } \\
\text { effectively communicate } \\
\text { information of competency of } \\
\text { health personnel administering } \\
\text { the vaccine and of equipment } \\
\text { sterility }\end{array}$ & $\begin{array}{l}\text { Selection bias of highly educated } \\
\text { parents limits generalizability }\end{array}$ \\
\hline [12] & $\begin{array}{l}\text { Hypothetical SLIV } \\
\text { program }\end{array}$ & $\begin{array}{l}\text { To describe parent and } \\
\text { student perspectives for } \\
\text { participation in SLIV }\end{array}$ & $\begin{array}{l}3 \text { middle and } 3 \text { high } \\
\text { schools in large, } \\
\text { urban school } \\
\text { district; Houston } \\
\text { independent school } \\
\text { district }\end{array}$ & $\begin{array}{l}\text { Survey of } 566 \\
\text { parent-student } \\
\text { dyads }\end{array}$ & $\begin{array}{l}\text { Survey } \\
\text { based on } \\
\text { focus groups }\end{array}$ & $\begin{array}{l}\text { Not applicable - no quantitative/ } \\
\text { statistical analysis }\end{array}$ & $\begin{array}{l}\text { SLIV participation by parents is } \\
\text { impacted by equipment sterility, } \\
\text { universal access of vaccines for } \\
\text { all students, and cost }\end{array}$ & $\begin{array}{l}1 \text { large, urban school district } \\
\text { limits generalizability; low } \\
\text { response rate and potential } \\
\text { selection bias; responses to } \\
\text { hypothetical program do not } \\
\text { reflect likelihood of action }\end{array}$ \\
\hline [29] & $\begin{array}{l}3 \text { school-located } \\
\text { vaccination } \\
\text { campaigns for } \\
\text { multiple vaccines, } \\
\text { including influenza } \\
\text { vaccine, and } \\
\text { delivered by } \\
\text { researchers and } \\
\text { local hospital }\end{array}$ & $\begin{array}{l}\text { To determine parental } \\
\text { trust and effect of trust- } \\
\text { building interventions in } \\
\text { school-located } \\
\text { vaccination, including } \\
\text { influenza vaccination }\end{array}$ & $\begin{array}{l}8 \text { middle schools in } \\
\text { a large, low- } \\
\text { income, urban } \\
\text { school district in } \\
\text { Texas }\end{array}$ & $\begin{array}{l}\text { Survey based } \\
\text { on trust } \\
\text { measures [36] } \\
\text { of } 1608 \text { parents } \\
\text { (year 1); } 844 \\
\text { parents (year 2) }\end{array}$ & $\begin{array}{l}\text { Trust survey } \\
\text { adapted } \\
\text { from Dugan } \\
\text { et al. [36] }\end{array}$ & $\begin{array}{l}\text { Annual household income, } \\
\text { survey language version, } \\
\text { participation in a previous SLIV, } \\
\text { child's health insurance status, } \\
\text { and perceived vaccine } \\
\text { importance were significantly } \\
\text { associated with parental trust in } \\
\text { SLIV (multiple linear regression } \\
\text { analysis; } R^{2}: 0.06, p<0.001 \text { ) }\end{array}$ & $\begin{array}{l}\text { Baseline trust in SLV was } \\
\text { moderately high among low- } \\
\text { income parents; higher trust and } \\
\text { participation can be attained by } \\
\text { increasing parents' perception of } \\
\text { vaccine importance }\end{array}$ & $\begin{array}{l}\text { Low response rate and } \\
\text { nonresponse bias; cannot } \\
\text { validate responses from self- } \\
\text { reported data }\end{array}$ \\
\hline
\end{tabular}


process [27]. Middleman et al. held focus groups of elementary, middle, and high school parents in a large urban school district of Houston, Texas and found that parental attitudes to SLIV were impacted by safety and trust issues regarding vaccines in general; programs should effectively communicate information regarding competency of health personnel administering the vaccine and equipment sterility [28]. In a related study, Middleman et al. conducted a survey of parent-student dyads in a large urban Houston school district; authors found that parental participation in SLIV was impacted by perceptions of equipment sterility, universal access of vaccines for all students, and cost [12]. Lastly, Won et al. conducted a 2-year survey of middle school parents in a low-income urban school district and found that baseline trust in SLIV programs was moderately high among low-income parents, while higher trust and participation of SLIV may be attained by increasing parental perception of vaccine importance [29].

\subsection{Facilitators}

The facilitators of parental attitudes and beliefs toward SLIV in the United States are illustrated in Table 2 and described below.

Cost: Parents were willing to participate in SLIV if they had no additional out-of-pocket expenses [9]. Free or low cost vaccines were significant facilitators of parental acceptance [28] but were less important when compared to other factors [12].

Vaccine efficacy: Parents with higher belief in vaccine efficacy were inclined to participate in SLIV [26].

Influenza severity: Parents with higher perceived severity of adolescent illness, including influenza, were more likely to accept SLIV [24]. Perceived severity of influenza illness was a predicting factor for parental consent [23].

Influenza illness susceptibility: Parental belief of their child being susceptible to influenza was a predicting factor of SLIV consent [23] and associated with acceptance if vaccines were offered for free [9]. Parents who had worried about the H1N1 virus in 2009 were also more likely to consent to SLIV participation [11].

Vaccine benefits: Parents with higher perceived benefit of influenza vaccine protecting against illness [23], combined with stronger belief in vaccination as a social norm [24] were more inclined to accept SLIV. The belief in vaccine benefit was also associated with acceptance if vaccines were offered free of cost [9].

Vaccine importance: Parental perception of vaccine importance was directly correlated with acceptance and trust in SLIV $[25,29]$.

Vaccination as a social norm: Social norms were associated with parental acceptance of school-located vaccination in general and for influenza vaccine specifically when compared to other adolescent vaccines [24]. Parental belief in vaccination as a social norm was associated with acceptance of SLIV if the vaccine was offered for free [9].

Influenza vaccine does not cause influenza: Parental belief in influenza vaccine not causing influenza was associated with acceptance of SLIV if the vaccine was offered for free [9].

Medical setting barriers: Endorsement of medical setting barriers such as inconvenience and time constraints promoted SLIV acceptance [9].

School setting advantages: Parents perceiving school-located vaccinations as convenient also facilitated SLIV acceptance [11,26-28].

Parental presence during vaccination: Flexible vaccination scheduling, such as during evenings or weekends, allowing parents to accompany children increased likelihood of SLIV participation [28].

Discussion with health care provider: Positive discussion about influenza vaccination and advice from a health care provider promoted parental consent and participation [9].
Trust in school health personnel: Having knowledge of credentials and having trust in the competency of health personnel administering vaccines improved parental consent [28].

Universal vaccine access in school: Ensuring availability of influenza vaccines for all students was an important factor for parental acceptance-more important than offering free or low cost vaccines [12].

\subsection{Barriers}

The barriers of parental attitudes and beliefs toward SLIV in the United States are illustrated in Table 3 and described below.

Cost: Parents were less likely to participate in SLIV due to cost [25,26] especially with multiple children in the household [9], however, it was not a primary concern when compared to other barriers [28].

Vaccine safety: Parental concerns of vaccine safety in general, including influenza vaccine in particular $[9,23,26]$, and risks [11] lowered their support to participate in SLIV.

Equipment sterility: Negative perceptions regarding sterility of equipment used for vaccine administration in a school setting was a significant factor impacting parental decision to trust and participate in SLIV [12].

Vaccine efficacy: Parents concerned with vaccine efficacy were less willing to participate in SLIV [9].

Influenza non-susceptibility: Parents with belief that their children were not susceptible to influenza were less likely to participate in SLIV [9].

Adverse effects: Parents concerned of vaccine side effects were less likely to consent to SLIV [23,27], with common concerns involving adverse effects of the live-attenuated influenza vaccine [7].

Influenza illness acquisition from vaccine: Parental concerns regarding influenza illness acquisition from the influenza vaccine was a barrier to SLIV participation [7].

Medical setting advantages: Parents preferred a medical setting for vaccination due to trust and safety issues regarding the child's well-being [26,27], potential side effects, and for proper vaccine administration $[11,23,28]$.

School setting barriers: Parental consent and acceptance of school vaccine delivery involved concerns regarding competency of person delivering the vaccine [9], the lengthy consent process [27], disorganization of the school [25], and the inability to address potential medical issues [28].

Parental absence during vaccination: Parents wanting to be present during the child's vaccination were less inclined to consent for SLIV in their absence $[9,23,26]$. Parents who felt that their children would want them present during vaccination was also a notable barrier [25].

Discussion with health care provider: Receiving negative physician advice based on incorrect contraindications of the liveattenuated influenza vaccine deterred parental participation in SLIV [7].

Distrust of vaccines and vaccination programs: Parents expressing skepticism of the influenza vaccine and/or the schoollocated vaccination program opted to either vaccinate their children through primary care physicians and pharmacies, or forgo influenza vaccination entirely. Negative attitudes toward the university-implemented vaccination program and associated misperceptions of research being performed on their children (i.e. to test an experimental vaccine) was a distinct barrier to SLIV participation [27].

Health insurance information: Parents were unwilling to provide health insurance information for billing, acting as a barrier to SLIV participation [26]. 
Table 2

Facilitators. Facilitators of parental attitudes and beliefs toward school-located influenza vaccination (SLIV) in the United States.

\begin{tabular}{|c|c|c|}
\hline Promoting factor & Description & Study \\
\hline Cost & Offering free/low cost vaccines & {$[9,12,28]$} \\
\hline Vaccine efficacy & Belief in vaccine efficacy & [26] \\
\hline Influenza severity & Belief in perceived severity of influenza & {$[23,24]$} \\
\hline $\begin{array}{l}\text { Influenza illness } \\
\text { susceptibility }\end{array}$ & $\begin{array}{l}\text { Parental belief in children being } \\
\text { susceptible to influenza and risk concerns } \\
\text { of H1N1 influenza }\end{array}$ & {$[9,11,23]$} \\
\hline Vaccine benefits & $\begin{array}{l}\text { Belief in benefit of influenza vaccine to } \\
\text { protect against influenza illness }\end{array}$ & {$[9,23,24]$} \\
\hline Vaccine importance & $\begin{array}{l}\text { Belief in importance of vaccination in } \\
\text { general }\end{array}$ & {$[25,29]$} \\
\hline $\begin{array}{l}\text { Vaccination is a } \\
\text { social norm }\end{array}$ & Belief that vaccination is a social norm & {$[9,24]$} \\
\hline $\begin{array}{l}\text { Influenza vaccine } \\
\text { does not cause } \\
\text { influenza }\end{array}$ & $\begin{array}{l}\text { Belief that the influenza vaccine does not } \\
\text { cause influenza }\end{array}$ & [9] \\
\hline $\begin{array}{l}\text { Medical setting } \\
\text { barriers }\end{array}$ & $\begin{array}{l}\text { Perception of inconvenience in accessing } \\
\text { regular medical settings for vaccination }\end{array}$ & [9] \\
\hline $\begin{array}{l}\text { School setting } \\
\text { advantages }\end{array}$ & $\begin{array}{l}\text { Perception of convenience in accessing } \\
\text { school setting for vaccination }\end{array}$ & $\begin{array}{l}{[11,26-} \\
28]\end{array}$ \\
\hline $\begin{array}{l}\text { Parental presence } \\
\text { during } \\
\text { vaccination }\end{array}$ & $\begin{array}{l}\text { Parents being present during vaccination } \\
\text { after school or during weekends }\end{array}$ & [28] \\
\hline $\begin{array}{l}\text { Discussion with } \\
\text { health care } \\
\text { provider }\end{array}$ & $\begin{array}{l}\text { Positive discussion with health care } \\
\text { provider about influenza vaccination }\end{array}$ & [9] \\
\hline $\begin{array}{l}\text { Trust in school health } \\
\text { personnel }\end{array}$ & $\begin{array}{l}\text { Trust in competency of health personnel } \\
\text { administering the influenza vaccine }\end{array}$ & [28] \\
\hline $\begin{array}{l}\text { Universal vaccine } \\
\text { access in school }\end{array}$ & $\begin{array}{l}\text { Access and availability of influenza } \\
\text { vaccine for all students in school }\end{array}$ & {$[12,28]$} \\
\hline
\end{tabular}

Health information privacy: Parents who were uncertain of the use/misuse of health information collected from their children's medical records were reluctant to consent to SLIV [27].

Pharmaceutical company: Poor communication and lack of knowledge regarding the pharmaceutical company manufacturing the influenza vaccine deterred parent participation in SLIV [28].

\section{Discussion}

\subsection{Facilitators}

Our review found that free or low cost vaccines generally facilitated parental acceptance of school-located influenza vaccination (SLIV) $[9,12,28]$. Parental acceptance is likely to be further facilitated by the Affordable Care Act [30] of 2010 which requires influenza (and other vaccines) to be covered by health insurance without charging a copayment or coinsurance, and the uninsured rate has declined by $43 \%$ from $16.0 \%$ in 2010 to $9.1 \%$ in 2015 [31]. Parents perceiving the convenience of a school setting over medical settings for vaccination were relatively more likely to consent $[9,11,26-28]$; having a positive discussion with a health care provider [9] and trusting the competency of health personnel administering the vaccine [28] significantly enhanced parental attitudes and acceptance for SLIV programs. Parents also preferred the scheduling of SLIV to take place after school or during weekends to allow parents the ability to accompany children during vaccination [28]. Additionally, the availability of influenza vaccines for all students was an important factor for parents [12,28].

Studies utilizing the Health Belief Model (HBM) [32] suggested that parents with enhanced perceptions of influenza susceptibility and severity, risks of H1N1 influenza, and benefits of influenza vaccination (including belief that the influenza vaccine does not cause influenza) were more likely to accept SLIV for their children $[9,23,24,26]$. Having beliefs in vaccine efficacy [26], vaccine importance $[25,29]$, and vaccination as a social norm $[9,24]$ also
Table 3

Barriers. Barriers of parental attitudes and beliefs toward school-located influenza vaccination (SLIV) in the United States.

\begin{tabular}{|c|c|c|}
\hline Barrier & Description & Study \\
\hline Cost & $\begin{array}{l}\text { Concerns of potential billing } \\
\text { related to school-located } \\
\text { vaccination }\end{array}$ & {$[9,25,26,28]$} \\
\hline Vaccine safety & $\begin{array}{l}\text { Safety concerns of vaccines in } \\
\text { general, including the influenza } \\
\text { vaccine }\end{array}$ & {$[9,11,12,23,26,28]$} \\
\hline Equipment sterility & $\begin{array}{l}\text { Trust concerns of cleanliness and } \\
\text { sterility of equipment used for } \\
\text { vaccination }\end{array}$ & {$[12,28]$} \\
\hline Vaccine efficacy & Concerns of vaccine efficacy & [9] \\
\hline $\begin{array}{l}\text { Influenza non- } \\
\text { susceptibility }\end{array}$ & $\begin{array}{l}\text { Parental belief that their children } \\
\text { are not susceptible to influenza }\end{array}$ & [9] \\
\hline Adverse effects & $\begin{array}{l}\text { Concerns of adverse effects from } \\
\text { vaccination }\end{array}$ & {$[7,23,27]$} \\
\hline $\begin{array}{l}\text { Influenza illness } \\
\text { acquisition from } \\
\text { vaccine }\end{array}$ & $\begin{array}{l}\text { Concerns of acquisition of } \\
\text { influenza illness from influenza } \\
\text { vaccine }\end{array}$ & {$[7,26]$} \\
\hline $\begin{array}{l}\text { Medical setting } \\
\text { advantages }\end{array}$ & $\begin{array}{l}\text { Parents preferred vaccination at } \\
\text { regular medical settings for trust } \\
\text { and safety reasons }\end{array}$ & {$[11,23,26-28]$} \\
\hline $\begin{array}{l}\text { School setting } \\
\text { barriers }\end{array}$ & $\begin{array}{l}\text { Concerns regarding competency } \\
\text { of person administering the } \\
\text { vaccine, school disorganization, } \\
\text { and inability to address medical } \\
\text { issues }\end{array}$ & {$[9,25,27,28]$} \\
\hline $\begin{array}{l}\text { Parental absence } \\
\text { during } \\
\text { vaccination }\end{array}$ & $\begin{array}{l}\text { Parents did not want their } \\
\text { children to receive vaccinations in } \\
\text { their absence }\end{array}$ & {$[9,23,25,26]$} \\
\hline $\begin{array}{l}\text { Discussion with } \\
\text { health care } \\
\text { provider }\end{array}$ & $\begin{array}{l}\text { Negative physician advice based } \\
\text { on incorrect live-attenuated } \\
\text { influenza vaccine } \\
\text { contraindications and concerns of } \\
\text { vaccine virus transmission to } \\
\text { household members with health } \\
\text { issues such as asthma }\end{array}$ & [7] \\
\hline $\begin{array}{l}\text { Distrust of vaccines } \\
\text { and vaccination } \\
\text { programs }\end{array}$ & $\begin{array}{l}\text { Distrust and skepticism about the } \\
\text { vaccination program and vaccines } \\
\text { in general, including influenza } \\
\text { vaccine. }\end{array}$ & {$[27,28]$} \\
\hline $\begin{array}{l}\text { Health insurance } \\
\text { information }\end{array}$ & $\begin{array}{l}\text { Unwillingness of parents to } \\
\text { provide health insurance } \\
\text { information }\end{array}$ & {$[26]$} \\
\hline $\begin{array}{l}\text { Health information } \\
\text { privacy }\end{array}$ & $\begin{array}{l}\text { Privacy concerns of use/misuse of } \\
\text { collected medical information and } \\
\text { distrust of vaccination program }\end{array}$ & [27] \\
\hline $\begin{array}{l}\text { Pharmaceutical } \\
\text { company }\end{array}$ & $\begin{array}{l}\text { Lack of knowledge of } \\
\text { pharmaceutical company } \\
\text { manufacturing the influenza } \\
\text { vaccine }\end{array}$ & {$[28]$} \\
\hline
\end{tabular}

promoted SLIV acceptance among parents. While most parents accepting of vaccines also consented to SLIV, some parents with no intention of vaccinating for influenza also stated willingness to participate if SLIV became available $[9,24]$.

\subsection{Barriers}

Significant barriers to SLIV acceptance were often related to the elements of the influenza vaccine, including concerns regarding vaccine safety $[9,11,12,23,26,28]$, vaccine efficacy [9], vaccine adverse effects $[7,23,27]$, and the risk of influenza acquisition from the vaccine itself [7].

Parental distrust of the school-located vaccination program was a notable barrier to participation, particularly for SLIV implemented by an external entity in a school setting without a health clinic [27]. Vaccine trust issues involved skeptical attitudes toward the vaccine $[11,27,28]$, concerns regarding equipment sterility and cleanliness of the school location $[12,28]$, and lacking knowledge of the pharmaceutical company that manufactured the vaccine [28]. 
Parents were unwilling to provide health insurance information for billing [26], and due to distrust in the vaccination program, parents felt uncertain regarding the use/misuse of health information collected from medical records of their children [27].

Trust issues, safety concerns, and medical setting advantages presented barriers for vaccination within a school setting $[11,23,26-28]$. Common concerns involved competency of health personnel administering the vaccine and their ability to address potential medical issues in a school setting $[9,25,27,28]$; many parents did not want their children to receive vaccination in their absence $[9,23,25,26]$. Other barriers included parental belief that their children were not susceptible to influenza [9] and having received physician advice that negatively portrayed liveattenuated influenza vaccination due to an incorrect understanding of contraindications [7]. Lastly, vaccine cost was generally perceived as a minor barrier for parents [9,25,26,28].

\subsection{School-located influenza vaccination in school-based clinics versus delivery by external agencies}

The studies included in this systematic review assessed parental attitudes and beliefs in relation to hypothetical SLIV scenarios as well as pilot program contexts. The pilot studies summarized here utilized external agencies such as health departments [7,23,26], university research staff [27], and hospitals [29] to deliver influenza vaccination in schools, as opposed to utilizing a school-based health clinic that is offered year-round; these two scenarios may present different issues of trust and concern among parents. Due to considerable heterogeneity in the format of schoollocated vaccination programs [25], future SLIV programs should take various scenarios into consideration during planning phases.

\subsection{Limitations}

Studies in this review reported limitations of low response rates [7,11,12,23,24,29], limited generalizability [9,11,12,23,24,26-29], and potential selection bias [12,23-25,28]. Some studies were geared toward hypothetical SLIV programs in the future $[11,12,25]$, and thereby, the responses of parents were based on potential action rather than actual behavior.

Differences in survey development, analysis, and subjective interpretation of qualitative responses of parents by authors limited comparability across studies as well as prioritization of parental barriers and facilitators. However, study findings encompass diversely varied populations and geographic regions within the United States which provides collective insight for potential prioritization within specific communities.

While the review of literature in this study is from 1990 to 2016, publication dates of reviewed articles span from 2007 to 2015 , with only two studies conducted before the 2009 H1N1 influenza pandemic. Thus, the analysis timeline of this systematic review may be biased toward studies after the 2009 H1N1 pandemic and possibly reflect elevated awareness of influenza among parental attitudes and beliefs toward SLIV programs. Additionally this may be reflective of the nature of discourse surrounding recent utilization of school-located immunization programs, signifying a young and evolving concept and area which necessitates further study.

\subsection{Public health implications}

Effective from the 2010-2011 influenza season, the ACIP recommends seasonal influenza vaccination annually for individuals aged 6 months and older without contraindications to prevent and control seasonal and pandemic influenza [33]. The Healthy People 2020 initiative includes the target of influenza vaccination coverage of 70\% [18]. Yet, influenza vaccination coverage in the general population was below par, ranging from $36.8 \%$ in Nevada to 56.6\% in South Dakota during the 2015-2016 influenza season, with a national vaccination coverage among children (6 months17 years) of $59.3 \%$ [34]. In this systematic review, we identified the facilitators and barriers of parental attitudes and beliefs toward SLIV for children in the United States that can assist in improving coverage and effectiveness of SLV programs. Specifically, influenza vaccination coverage is improved among children whose parents did not plan to vaccinate in the absence of a school-located program $[9,24]$. Further, improving influenza vaccination coverage among school children in general improves herd immunity in the total population. The Affordable Care Act [30] of 2010 lowered the uninsured rate by $43 \%$ from $16.0 \%$ in 2010 to $9.1 \%$ in 2015 [31], and health insurance now covers influenza vaccines without additional out-of-pocket payments. While cost has become a lesser barrier, SLV programs can facilitate improved access to influenza vaccination for school-aged children.

\subsection{Systems thinking in school-located influenza vaccination}

Health program strategies based on systems thinking focus on an ongoing iterative learning of systems understanding, analysis and improvement, and leadership and collaboration across disciplines, sectors, and organizations [35]. School-located influenza vaccinations are collaborative programs between health and education sectors with great potential for improving influenza vaccination coverage among school-aged children. SLIV programs directly benefit vaccinated children who express protective immune response, as well as indirectly benefiting the larger community by reducing transmission pathways. We identified facilitators and barriers of parental attitudes and beliefs toward SLIV from a systems thinking perspective. Through systematic understanding, analysis, and identification of facilitators and barriers, this study provides evidence to improve the design and implementation of current and future SLIV programs by leveraging key promoting factors and addressing potential barriers.

\section{Funding}

This study is supported by NIH/NIGMS R01GM109718 and NSF/ NRT 1545362; the funding sources had no role in study design; collection, analysis, and interpretation of data; writing of the paper; or the decision to submit it for publication.

\section{References}

[1] Middleman A. School-located vaccination for adolescents: past, present, and future and implications for HPV vaccine delivery. Hum Vaccin Immunother 2016:12:1599-605.

[2] Hull HF, Ambrose CS. Current experience with school-located influenza vaccination programs in the United States. Hum Vaccin 2011;7:153-60.

[3] Ogilvie G, Anderson M, Marra F, McNeil S, Pielak K, Dawar M, et al. A population-based evaluation of a publicly funded, school-based HPV vaccine program in British Columbia, Canada: parental factors associated with HPV vaccine receipt. PLoS Med 2010;7:e1000270.

[4] Russell M, Raheja V, Jaiyesimi R. Human papillomavirus vaccination in adolescence. Perspect Public Health 2013;133:320-4.

[5] Brotherton JML, Deeks SL, Campbell-Lloyd S, Misrachi A, Passaris I, Peterson K, et al. Interim estimates of human papillomavirus vaccination coverage in the school-based program in Australia. Commun Dis Intell Q Rep 2008;32:457-61.

[6] Effler PV, Chu C, He H, Gaynor K, Sakamoto S, Nagao M, et al. Statewide schoollocated influenza vaccination program for children 5-13 years of age, Hawaii, USA. Emerg Infect Dis 2010;16:244-50.

[7] Carpenter LR, Lott J, Lawson BM, Hall S, Craig AS, Schaffner W, et al. Mass distribution of free, intranasally administered influenza vaccine in a public school system. Pediatrics 2007;120:e172-8.

[8] King Jr JC, Stoddard JJ, Gaglani MJ, Moore KA, Magder L, McClure E, et al. Effectiveness of school-based influenza vaccination. $N$ Engl J Med 2006;355:2523-32. 
[9] Allison MA, Reyes M, Young P, Calame L, Sheng X, Weng H-YC, et al. Parental attitudes about influenza immunization and school-based immunization for school-aged children. Pediatr Infect Dis J 2010;29:751-5.

[10] Middleman AB, Tung JS. Urban middle school parent perspectives: the vaccines they are willing to have their children receive using school-based immunization programs. J Adoles Health 2010;47:249-53.

[11] Brown DS, Arnold SE, Asay G, Lorick SA, Cho B-H, Basurto-Davila R, et al. Parent attitudes about school-located influenza vaccination clinics. Vaccine 2014;32:1043-8.

[12] Middleman AB, Short MB, Doak JS. School-located influenza immunization programs: factors important to parents and students. Vaccine 2012;30:4993-9.

[13] Tran CH, Sugimoto JD, Pulliam JRC, Ryan KA, Myers PD, Castleman JB, et al. School-located influenza vaccination reduces community risk for influenza and influenza-like illness emergency care visits. PLoS One 2014;9:e114479.

[14] Pannaraj PS, Wang H-L, Rivas H, Wiryawan H, Smit M, Green N, et al. Schoollocated influenza vaccination decreases laboratory-confirmed influenza and improves school attendance. Clin Infect Dis 2014;59:325-32.

[15] Weycker D, Edelsberg J, Halloran ME, Longini Jr IM, Nizam A, Ciuryla V, et al. Population-wide benefits of routine vaccination of children against influenza. Vaccine 2005;23:1284-93.

[16] Centers for Disease Control and Prevention (CDC). Update on influenza A (H1N1) 2009 monovalent vaccines. MMWR Morb Mortal Wkly Rep 2009;58:1100-1.

[17] Flu Vaccination Coverage, United States, 2015-16 Influenza Season । FluVaxView | Seasonal Influenza (Flu) | CDC. <https://www.cdc.gov/flu fluvaxview/coverage-1516estimates.htm>; n.d. [accessed February 21, 2017].

[18] Healthy People 2020. <https://www.healthypeople.gov/>; n.d. [accessed February 21, 2017].

[19] Piedra PA, Gaglani MJ, Kozinetz CA, Herschler G, Riggs M, Griffith M, et al. Herd immunity in adults against influenza-related illnesses with use of the trivalent-live attenuated influenza vaccine (CAIV-T) in children. Vaccine 2005;23:1540-8.

[20] Cummings GE, Ruff E, Guthrie SH, Hoffmaster MA, Leitch LL, King Jr JC Successful use of volunteers to conduct school-located mass influenza vaccination clinics. Pediatrics 2012;129(Suppl 2):S88-95.

[21] King Jr JC, Lichenstein R, Cummings GE, Magder LS. Impact of influenza vaccination of schoolchildren on medical outcomes among all residents of Maryland. Vaccine 2010;28:7737-42.

[22] Moher D, Liberati A, Tetzlaff J, Altman DG, PRISMA Group. Preferred reporting items for systematic reviews and meta-analyses: the PRISMA statement. PLoS Med 2009;6:e1000097.
[23] Cheung S, Wang H-L, Mascola L, El Amin AN, Pannaraj PS. Parental perceptions and predictors of consent for school-located influenza vaccination in urban elementary school children in the United States. Influenza Other Respir Viruses 2015;9:255-62.

[24] Gargano LM, Weiss P, Underwood NL, Seib K, Sales JM, Vogt TM, et al. Schoollocated vaccination clinics for adolescents: correlates of acceptance among parents. J Commun Health 2014;40:660-9.

[25] Kelminson K, Saville A, Seewald L, Stokley S, Dickinson LM, Daley MF, et al. Parental views of school-located delivery of adolescent vaccines. J Adoles Health 2012;51:190-6.

[26] Kempe A, Daley MF, Pyrzanowski J, Vogt TM, Campagna EJ, Dickinson LM, et al. School-located influenza vaccination with third-party billing: what do parents think? Acad Pediatr 2014;14:241-8.

[27] Herbert NL, Gargano LM, Painter JE, Sales JM, Morfaw C, Murray D, et al. Understanding reasons for participating in a school-based influenza vaccination program and decision-making dynamics among adolescents and parents. Health Educ Res 2013;28:663-72.

[28] Middleman AB, Short MB, Doak JS. Focusing on flu: parent perspectives on school-located immunization programs for influenza vaccine. Hum Vaccin Immunother 2012;8:1395-400.

[29] Won TL, Middleman AB, Auslander BA, Short MB. Trust and a school-located immunization program. J Adoles Health 2015;56:S33-9.

[30] About the Law. HHS.gov 2013. <https://www.hhs.gov/healthcare/about-thelaw/>; 2013 [accessed February 21, 2017].

[31] Obama B. United States health care reform: progress to date and next steps. JAMA 2016:316:525-32.

[32] Janz NK, Champion VL, Strecher VJ. The health belief model. In: Glanz K, Rimer BK, Lewis FM, editors. Health behavior and health education: theory, research, and practice. San Francisco, CA: John Wiley and Sons, Inc.; 2002. p. 45-66.

[33] CDC - ACIP - Advisory Committee on Immunization Practices (ACIP) Home Page - Vaccines. <http://www.cdc.gov/vaccines/acip/>; n.d. [accessed July 3, 2015].

[34] 2015-16 Influenza Season Vaccination Coverage Report | FluVaxView | Seasonal Influenza (Flu) | CDC. <https://www.cdc.gov/flu/fluvaxview/ reportshtml/reporti1516/reporti/index.html>; n.d. [accessed February 21, 2017]

[35] Swanson RC, Cattaneo A, Bradley E, Chunharas S, Atun R, Abbas KM, et al. Rethinking health systems strengthening: key systems thinking tools and strategies for transformational change. Health Policy Plan 2012;27(Suppl. 4): iv54-61.

[36] Dugan E, Trachtenberg F, Hall MA. Development of abbreviated measures to assess patient trust in a physician, a health insurer, and the medical profession. BMC Health Serv Res 2005;5:64. 